\title{
Research on Big Data Application in Smart Education System
}

\author{
Zhifeng $\mathrm{Hu}$ \\ Wuhan Business University, Wuhan, Hubei, 430056 \\ hzf@wbu.edu.cn
}

Keywords: Big Data. Smart Education. Informatization.

Abstract. Smart education system, an advanced form of modern educational informatization supported by technologies like cloud computing, Internet of things and mobile Internet, builds ubiquitous network environment, cloud computing data center, sensing system of multidimensional Internet of things and all kinds of application systems on the basis of which a series of information services are launched. Application of big data is the focus and challenge of smart education system. This article carries out research on the application of big data in smart education system, promoting the transformation of information portals of universities into service portals.

\section{Introduction}

Information construction of universities has entered the stage of smart education, as manifested by the rapid popularization of technologies such as mass storage, cloud computing and Internet of things in universities. The application of big data will also become one of the core applications of smart education system. Big data application connects the physical platform for data storage and computation, the supporting platform for information collection and sorting, and the management platform for data analysis and processing. Data connects all parts of smart education system. The vast amount of information on the status and activities of each part pooled together will be organized through big data analysis to present their patterns of development and put into smart application [1] .

\section{Smart Education System.}

Information Standard System. The construction of smart education system in campus is an informatization project integrating unification of management, mechanism and technology. Management is the source of control, business is the source of service, data is the source of basis, and information standard is the source of guidance. Information standards, the foundation of informatization construction in campus, are a necessary condition to realize application service, data sharing and information exchange service [2] .

Design and implementation of information standards in universities should follow the normative mode. In accordance with Information Standards for Education Management---Informatization Standards for Higher Education Institutions and drawing from the successful experiences of standardization work at home and abroad, normative systems and information standards of universities shall be designed and compiled based on their actual needs and by following the "Five Uniformization" principle of "uniformize file formats", "uniformize index systems", "uniformize terminologies", "uniformize classification codes" and "uniformize information exchange formats".

System for safe operation and maintenance of informationization. The construction of security maintenance system, an important support for the construction of smart education system, involves physical security, operation security and information security. Physical security includes environmental security, equipment security, line security, disaster prevention and recovery. Operation security includes management security, application security, risk analysis and assessment, auditing and tracking, backup and recovery. Information security includes operating system and application system security, network security, data and communication security, virus and Trojan prevention, encryption, identity authentication and access control [3]. 
Network service system. The basis of the construction of smart education system is the comprehensive integration of networks, which means to integrate various types of networks on campus, adopt unified management and control, and provide diversified network accesses and open standard interfaces, so as to ensure the network and communication for different application services within the smart campus system. Introducing cloud computing and other services to smart campuses and providing private cloud computing services to universities. By providing the teachers and students with a variety of personalized information services, cloud services can be effectively used for teaching, scientific research, management and living purposes, allowing them to enjoy the convenience of cloud computing services. Building a central data platform. By building a unified data sharing and interaction center, uniformization of information portal, identity authentication and single sign on can be realized [4] .

Public service system. Application service: Providing business service modules, including management and service modules such as educational administration, scientific research, OA, finance, student affairs, recruitment, personnel and logistics.

General information service: Providing public service modules, in particular a variety of service functions including user management, business functions, authorization, log audit, identity authentication, reports, data exchange, inquiry statistics, workflow, information, institutional framework and network community [5] .

Data resource system. As a data center, data resource system, which is a basic data layer for the overall informatization of different departments in universities, includes application business data, application exchange data and other core contents.

Data resource layer can, by integrating multiple data sources, provide universities with comprehensive and accurate data, support data exchange between systems, provide a unified authentication service, and provide a unified and sharing data support platform [6].

Establishing a shared data center based on big data technology. The shared data center based on SOA system architecture can, by providing uniform, standard data interfaces, realize unified management and use of data. With the support of big data technology, the data will be more effectively and accurately transmitted to the top level application system, enabling more inclusive, accurate, intelligent and optimized smart education applications.

Comprehensive information service system. The comprehensive information service system is operated on the data storage and exchange platform. Based on the application of big data and from the perspective of the whole university, the system carries out on this platform centralized cleaning, conversion and distributed storage of data collected from all business systems, before unified external data service is provided. In the form of reports or charts, in-depth statistical analysis is provided. This provides strong support for decision-making of all kinds of managing staff in universities by providing accurate and effective comprehensive system for school situation inquiry, report generation system and information filling system.

The comprehensive information service system is based on big data platform. big data platform on campus, based on the data integration, storage, mining and analysis of big data technology, provides top layer application system with data support service.

\section{The application of big data in smart education system.}

The concept of big data. Big data, a product of high-tech age that emphasizes the capture, management, and processing of data, is not a single technology. It can be understood as a huge amount of informational assets with diverse sources, or an agglomeration of data which cannot be easily captured, stored, managed and analyzed by general software. Integrated and analyzed with a new approach, big data can discover valuable information, contribute to decision making, and create new value $[7,8]$.

Collection and management of big data. The various types of structured, semi-structured (or weakly structured) and unstructured massive data, such as radio frequency data obtained through 
RFID, sensor data, social network interaction data and mobile Internet data are the fundamental of big data service models.

Big data management means storing collected data in the memory, establish corresponding database and then utilize and manage the data. . The focus of big data management is to address the management and processing technologies of complex structured semi-structured and unstructured big data. Key problems to be solved include the availability of storage, expression, management, reliability and effective transmission of big data. We should develop reliable distributed file system (DFS), storage with optimization of energy efficiency, computing-integrated storage, big data redundancy and big data storage technologies with high efficiencies and low costs. We should make a breakthrough in distributed and non-relational big data management and processing technology, data fusion technology of heterogeneous data, data organization technology, and conduct researches on big data modeling technology. We should also make a breakthrough in the transfer, backup, replication and other technologies of big data and develop visualization technologies of big data. Data storage can be accomplished through storage devices in smart campus data center, and data management and handling can be realized through the central database of data center $[9,10]$.

The basic data of big data system is mainly from the existing information management system of universities. The main function of big data system is to collect, analyze and display the basic data. Besides various types of third party management systems, the big data system collects and completes information on users on campus from WEB and mobile APP, like information on geographic location and notices.

Application of big data in smart education system. Big data analysis technology can provide strong data analysis for student affairs management. From the point of view of data mining tasks and methods, the primary objective in the application of big data in smart education system is the visualization of data, which allows users to observe data processing results directly. The second objective is to provide predictive analysis, giving prospective judgment at a certain degree according to visualized data.

Big data technology can, by mining the information and knowledge hidden in a vast amount of data and providing the basis for social and economic activities of human beings, improve operational efficiency. Big data analysis and mining technologies can also be applied to improve teaching quality in universities, analyze behaviors of users in campus network and public opinions, which will effectively promote the in-depth integration of information technology and teaching.

Promoting the optimization of teaching results. Data resources of university include abundant information on teaching, which can be effectively used. By analyzing and mining the data such as click rate, download, and repetition rate of knowledge points, the emphasis and difficulty of curriculum can be obtained, personalized learning guidance can be developed according to the visit behaviors of individual student, and guidance can also be made on the career development of students in future.

Effective asset management. By completing the registration and marking of asset information such as building information (the name of the building, construction area, number of floors, number of rooms on each floor, total number of beds, fire control facilities and monitoring facilities), condition of living (name of school, profession, number and grade of students), and property information (hardware and software facilities), the administrators can inquire and manage information about people, finance and assets in the management system in accordance with their access level by using fixed keyword inquiry and user-defined fuzzy inquiry, realizing the effective management of assets.

User behavior analysis. Activities trajectory information in the campus network is organized and stored. All kinds of activity information collected are organized according to user types and time before the activity trajectory information is generated and stored. Through the analysis of big data, an early warning can be made for the abnormality in the activities of a specified object. Besides, the administrators can make inquiries on personnel, affairs and services, early warning information and emergencies.

Decision-making aids. Focusing on data capturing smart decision analysis service application is built. By building the shared database with "captured data", the authority and consistency of data used 
by all systems are guaranteed. By ensuring the consistency, integrity and accuracy of data of all business systems in the universities with enterprise level information standards, accessibility, intelligibility, reliability and availability of basic data throughout the universities are enhanced in data quality.

With big data, statistical summaries can be made on various data, including population statistics, event statistics and department statistics and the like, which can be displayed in the form of reports and charts (histograms, pie charts). Big data supports decision making process by allowing administrators to clearly see the statistics on people and events of various departments under their jurisdictions.

Analysis of public opinions. Public opinions of universities in network age are mainly shared on campus BBS, micro-blogs, WeChat and all kinds of instant communication software. Against the background of big data technology, a typical application of big data is to accurately and quickly grasp the trend of online public opinions and guide students to express their views properly.

\section{Conclusions}

With the increase of user number and data on user behavior in campus network, big data application can enhance the value of massive data and provide the predictability and relevance for administration work. The application of big data, by providing more intelligent, efficient and accurate services for teaching, scientific research, management and living, can promote the in-depth integration of information technology and education and realize the overall improvement of the level of education. With cloud computing and IoT serving as the network basis for the collection, distribution, exchange and storage of information, and big data as the core technology for data mining and analysis, a smart education system featuring information collection, resource sharing, application integration and comprehensive operation can be built to meet the increasing demands at all levels in campus development. On the unified smart education platform, highly efficient campus management and intelligent teaching process can be realized to improve the well-being of teachers and students on campus.

\section{References}

[1] Dongxing Jiang.et al: Journal of East China Normal University. No S1(2015),p.119-125. (In Chinese)

[2] Junfeng Luo.et al: The Chinese Jourmal of ICT in Education. No 3(2014),p.11-13. (In Chinese)

[3] Qian Lu: Computer Science.Vol.38(2011),p.18-21. (In Chinese)

[4] Qing-bin Sang: Journal of Nantong Textile Vocational Technology College.Vol.13(2013), p.84-87. (In Chinese)

[5] Jing-yan Zhao.et al: Information Science. Vol.34(2016) ,p.92-95. (In Chinese)

[6] CHENG Xue-Qi.et al: Journal of Software, Vol.25(2014), p.1889-1908. (In Chinese)

[7] Mayer-Schönberger V, Cukier K. Big data: A revolution that will transform how we live, work, and think. Houghton Mifflin Harcourt ( 2013 )

[8] Arasu A,Chaudhuri S,Chen Z,et al:IEEE Data Engineering Bulletin,35-2(2012),P.14-23

[9] Labrinides A,Jagadish HV:PVLDB,5-12(2012),P.2032-2033

[10] White T. Hadoop: The definitive guide. O'Reilly Media, Inc. (2012) 